\title{
(RE)CAPACITAR SOBRE LA (DIS)CAPACIDAD: LAS PERSONAS DISCAPACITADAS COMO CIUDADANAS
}

\author{
ROSA MARÍA ALEMANY \\ MONTSERRAT MESTRES \\ ELISABET TEJERO \\ Profesoras de la Escola Universitària de Treball Social de la Universitat de Barcelona.
}

\section{ABSTRACT}

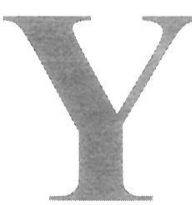

a hace tiempo que las familias de las personas con discapacidad psíquica hacen saber a la Administración Pública su preocupación entorno a las necesidades que los jóvenes y adultos tienen para poder emanciparse del hogar familiar. El envejecimiento de los padres, las difi-

cultades de acogida de otros familiares y la complejidad de atención que generan ciertas discapacidades psíquicas son, entre otros aspectos, razones que están alimentando esta preocupación.

Como equipo de profesoras de la Escola Universitària de Treball Social de Barcelona con experiencia profesional en la atención a las personas con discapacidad, nos ha parecido interesante y oportuno repensar las dimensiones sociopolíticas de esta situación siguiendo una línea de reflexión ligada a las políticas de igualdad de oportunidades y de extensión de la ciudadanía. Presentamos por ello un conjunto de reflexiones en torno a "la igualdad de oportunidades para los colectivos de personas con alguna discapacidad".

Claves conceptuales: paradigma relacional, calidad de vida, ciudadanía.

\section{INTRODUCCIÓN}

La incorporación de las personas con disminución a los ritmos y formas de vida cotidiana de la sociedad actual forma parte de un extenso debate. En este sentido son muchas las reflexiones que se hacen sobre el papel que tienen las políticas sociales como mecanismos preventivos de los riesgos de exclusión, especialmente para aquellos colectivos considerados vulnerables en el contexto de la sociedad occidental. Tanto los discursos académicos como los discursos políticos centran especialmente sus reflexiones y propuestas en torno a los as- 
pectos vinculados a la ocupación. El trabajo remunerado como actividad socialmente reconocida es aún, sin duda, una fuente de sentido e identidad. Pero también es sabido que la búsqueda de visibilidad y utilidad social como personas, como ciudadanos, forma parte de un proceso de más amplio alcance.

La vivencia de la integración social, es decir, de sentirse legitimado para participar de los derechos y obligaciones del conjunto de la población más allá del ámbito productivo, se revela también como un factor de primer orden para el desarrollo integral de cualquier persona $y$, en especial, para aquellas personas que tienen alguna discapacidad. Las relaciones normalizadas entre las personas son un poderoso recurso para combatir las limitaciones que se dan en la vida cotidiana. Este hecho es aún más significativo si tenemos en cuenta el bagaje cultural de las diversas sociedades mediterráneas del cual participamos. En este sentido la dimensión relacional de la vida cotidiana puede poner en marcha dinámicas de cooperación, de intercambio, de redistribución y reciprocidad ejes básicos de las interacciones humanas y convertirlas en principios de orientación de las mismas.

La incorporación de estos valores a la política social se formula normalmente más como un desiderátum que como una práctica claramente aplicable a la realidad. No es extraño, por lo tanto, que esta situación se manifieste de forma persistente en toda la Europa comunitaria aun después de décadas de consolidación del estado de bienestar. En definitiva se trata de ir a contracorriente de muchos de los valores subyacentes en la economía de mercado y de la democracia parlamentaria y representativa. La política social, ciertamente, no puede actuar como un ámbito independiente y aislado del contexto político y económico que la cobija y es en esta línea que la introducción de innovaciones ligadas a la subjetividad se perfila como un proceso lento y complejo.

Una de las rupturas conceptuales que propone el paradigma relacional es la de dejar de considerar la independencia como una definición unívoca del bienestar personal y como finalidad prioritaria de la política social. El camino hacia la independencia individual, medida de éxito en la sociedad europea del bienestar, no parece que pueda encontrar vías reales de desarrollo si no se encuadra dentro de una comprensión más amplia de las relaciones sociales. Nos referimos a la conceptualización de la interdependencia como fuente básica de la relación humana, como una reflexión que desvela la necesidad de otros sujetos en el desarrollo de la propia vida, como una ruptura de la idea de la omnipotencia del individuo ante de su destino. Esta relación de interdependencia se expresa en función de los otros significativos, es decir, de personas relevantes que acompañan y participan en procesos 
personales desde formas absolutamente plurales. Sin duda la reivindicación teórica de esta forma de organización social y política no va exclusivamente dirigida a personas con alguna discapacidad, ni tampoco es nueva; en todo caso su novedad radica en la manera de articularse en cada circunstancia.

Así pues este artículo reflexiona sobre los aspectos procesuales y dinámicos que recogen el tránsito hacia la vida adulta de las personas con discapacidad psíquica y, en este sentido, toman especial protagonismo aspectos tales como las vías de emancipación de jóvenes y adultos y la red relacional y comunicativa de la vida cotidiana. Se trata, en definitiva, de una exploración sobre la dimensión vivencial y simbólica de las personas que aspiran a la inclusión social, al respeto de la comunidad en que viven y a la libertad como ciudadano con derechos y obligaciones en un contexto democrático y constitucional como el nuestro.

Para poder dar respuesta a estos intereses, nos ha parecido indispensable elaborar un marco de referencia teórico que, sin ánimo de saturar al lector, pueda introducir reflexiones muy actuales en torno a la integración social de las personas con alguna discapacidad. Estos análisis se hacen extensivos a todos aquellos colectivos que por diferentes circunstancias quedan relegados o, aún más, excluidos de los circuitos de derechos y bienestar de nuestra sociedad. De manera muy especial por parte de la Unión Europea y de las fundaciones y asociaciones de personas con discapacidades, se está luchando para formular unas directrices que permitan a Estados, Gobiernos y entidades públicas y privadas ejercer una función de liderazgo y acompañamiento en el proceso de producción de una ciudadanía plena para todos. Se trata de un reto que implica la labor nada fácil de cambiar formas de ver la realidad de nuestro entorno, formas de pensar y de actuar, en que las familias - todos y cada uno de sus miembros-, los políticos y los profesionales sean los protagonistas.

\section{LA PERSPECTIVA RELACIONAL: UN ANTÍDOTO CONTRA EL ESTIGMA DE LA DISCAPACIDAD}

La mirada social hacia aquello que se escapa del flujo de la normalidad más estandarizada ha generado y aún genera reacciones estigmatizadoras. Hasta hace unas décadas, los investigadores y profesionales de la cuestión social participaban en la producción de un saber muy crítico respecto a las dinámicas sociales que provocaban marginación o segregación. Se podría decir que desde una perspectiva europea existía una visión bastante unánime sobre la capacidad de las políticas sociales de los diferentes estados del bienestar para mitigar y 
erradicar muchas de las desigualdades sociales. Estas desigualdades eran, muy a menudo, interpretadas como un hecho externo al funcionamiento de los Estados y de las políticas sociales de carácter democrático. Aunque enfaticemos la confianza que la política de la igualdad y del bienestar supone para la mayoría de ciudadanos de nuestro país, ésta ha llegado a ser también objeto de valoraciones críticas, sobre todo en los últimos veinte años. Sin ánimo de privar de legitimidad filosoficopolítica al modelo del estado del bienestar, pensamos que se deben tomar en consideración algunas de estas críticas, especialmente cuando nos adentramos en el ámbito de la discapacidad psíquica.

No se está aludiendo a la tan traída y llevada crisis fiscal del Estado, sino a los efectos no deseados que ha provocado la propia política social con sus programas de integración. La cara oculta de la política de reducción de la desigualdad ha ido generando un entramado de clasificaciones administrativas que han acabado agrupando a los individuos especialmente vulnerables en una categoría estandarizada. Se trata de una situación que ha tenido importantes repercusiones en la comprensión del retraso mental y en la promoción de la integración social de las personas con discapacidad.

Las posibilidades que la sociedad otorgaba a estas personas para disfrutar o no de determinados espacios y oportunidades sociales estaba sometida al examen del cociente de inteligencia. La utilización de este tipo de cocientes fue, durante muchos años, la resultante de interpretar la discapacidad como una deficiencia que describía al individuo en su totalidad. Cualquier otro aspecto de su persona quedaba condicionado o anulado por esta valoración pretendidamente objetiva. Desde este punto de vista, la voluntad de afrontar el tratamiento social de la discapacidad constituyó, a la vez, una manera de reducir y constreñir a las personas al estrecho margen de una clasificación parcial. Como efecto no deseado, la política social colaboraba en la producción de un estigma basado en la imposibilidad de que las personas con discapacidad se desarrollen plenamente en la sociedad en la que viven. Es más, desde el punto de vista de la praxis médica quizá no tanto del discurso, las resistencias a admitir la discapacidad como un estado cambiante y dinámico están, todavía, muy arraigadas.

Romper con el esencialismo filosófico no ha sido una labor fácil ni rápida, y por esta razón ha hecho falta un importante esfuerzo pedagógico entre los propios profesionales que se han visto volcados a un más que significativo cambio de mentalidad. El concepto de conducta adaptativa, que llega desde la American Association on Mental Health (AAMRA) en los años sesenta, promueve un salto cualitativo en los paradigmas de comprensión sobre el retraso mental que rápidamente se incorporan a otros tipos de discapacidad. Tal como exponen 
Aiguabella y González (1997), la definición de la AAMRA es la que más se ha destacado, probablemente porque asume por un lado la naturaleza evolutiva de la conducta adaptativa como consecuencia de los cambios en las expectativas sociales para cada edad, y por otro lado tiene en cuenta la relación determinante del entorno cultural diferenciado en cada sociedad y las posibilidades que cada medio facilita a estos individuos en su desarrollo.

Empezar a pensar el espacio social de la discapacidad teniendo consciencia de aquello que supone el ciclo de vida abre un nuevo campo de intervención para producir bienestar. Es decir, tener en cuenta las múltiples transiciones biográficas por las que pasan las personas, entre ellas la emancipación del hogar de origen, permite dar un salto cualitativo en la política social. Ésta tiene, entonces, la capacidad de interpretar a las personas en clave de diversidad y de introducir la dimensión temporal y de cambio en la comprensión de los procesos personales y sociales.

De entre todas las innovaciones teóricas y metodológicas que genera este paradigma, destacaríamos dos de las premisas que, según nuestro punto de vista, constituyen la clave para replantear la intervención social en este campo. En primer lugar, el protagonista máximo de valoraciones, programas y servicios es la persona, y lo es en cuanto se concibe como un sujeto único, plural, complejo y cambiante. Éste no es un argumento para nada menospreciable en un contexto en que el mito de la cuantificación conlleva considerar a las personas como entidades estadísticas que se expresan bajo formulaciones numéricas. Reivindicar el retorno a la persona postura propia de una filosofía humanista nos conduce a valorar las necesidades y las capacidades humanas como principios orientadores de pensamiento y de acción. Ciertamente, hablamos de necesidades materiales, pero nos parece tanto o más importante subrallar cuestiones básicas de la condición humana, como el desarrollo de la emocionalidad, de la posibilidad de escoger y decidir sobre la propia vida, de la capacidad de expresarse libremente, de moverse, de comunicarse, de hacer y de crear. Como dice Hanna Arendt (1993), las personas persistimos en "hacer" y "construir". Siendo así, el retraso mental o cualquier otro tipo de discapacidad puede dejar de considerarse un rasgo definitorio de la persona para llegar a ser un "estado en movimiento". De esta manera, se pueden superar las tendencias devaluadoras del rol social de la persona con discapacidad (Coordinadora de Tallers de Catalunya, 1999).

Cabe decir que la defensa del valor de la individualidad no supone asumir una metodología de trabajo individualista, sino por el contrario: desarrollar una mirada intensiva al sujeto nos confronta con una realidad multidimensional forjada de relaciones sociales. Por esta razón, la 
segunda premisa a destacar es aquella que sitúa al individuo en continua interacción con el medio social que lo rodea. No hablamos únicamente de la familia, hablamos de la escuela, de los centros de formación y de trabajo, de la comunidad de vecinos y de amistades, del barrio, de la vida en la ciudad o en el pueblo.

Desde esta perspectiva no sería difícil dirigir el análisis hacia enfoques muy deterministas. Pero lo que aquí defendemos es una perspectiva relacional caracterizada por la vertiente ecológica y ambiental de las relaciones sociales (A.Angel, 1995). Nos referimos a la continua interdependencia que existe entre cualquier persona, el medio social en el que desarrolla su vida cotidiana y el conjunto de la sociedad. Los múltiples tipos de relaciones sociales que de ello se derivan son el producto de una construcción colectiva, en la que todos somos productores y receptores a la vez.

\section{LOS DERECHOS SOCIALES Y LA IGUALDAD DE OPOR- TUNIDADES PARA LAS PERSONAS CON DISCAPACIDAD.}

Sería osado por nuestra parte presentar a la sociedad como una construcción colectiva en la que las relaciones entre las personas se estructuran en función de un reparto equilibrado de poder y en la que las condiciones de partida son las mismas para todos, especialmente cuando tratamos la cuestión de la discapacidad. Al contrario: relaciones, sí y desiguales, también. En el contexto europeo ha sido necesario, y todavía lo es, defender una discriminación positiva para todas aquellas personas con discapacidad que se encuentran día a día con barreras políticas, económicas, sociales, culturales y físicas que les impiden sentirse ciudadanos de pleno derecho.

A modo de ilustración el Programa Helios II. Guía Europea de Buena Práctica. Hacia la Igualdad de Oportunidades para las personas discapacitadas de la Comisión Europea (1996) denuncia la siguiente situación: Con harta frecuencia se percibe la discapacidad como un problema propio de un individuo. En los últimos años, las personas discapacitadas han empezado a caer en la cuenta de que el término discapacidad disimula un sistema complejo de restricciones sociales... a pesar de las abundantes medidas e instrumentos de politica social desplegados en los últimos años, no se ha logrado situar a los ciudadanos europeos discapacitados en pie de igualdad en términos de derechos y responsabilidades. Y por ello es preciso fortalecer la condición humana reivindicando el valor universal de ser persona y sujeto de derechos y obligaciones. 
Desde la redacción de la Carta de los Derechos Humanos hasta el reconocimiento explícito de las constituciones políticas de los países de la Unión Europea sobre los derechos sociales, son muchas las contradicciones que legitiman una lucha positiva para incorporar a las personas con discapacidad en la dinámica del conjunto de la ciudadanía. Y en este sentido, no podemos separar la conquista de los derechos sociales de la persona de las formulaciones del concepto de ciudadanía. Es preciso recordar las aportaciones de T.H. Marshall que, en 1952, amplió los límites de los derechos políticos y económicos a su dimensión social: la ciudadanía social recoge el conjunto de derechos que van desde el derecho a un mínimo bienestar económico y de seguridad hasta el derecho a participar plenamente del patrimonio social y a vivir según unos niveles de vida dignos.

Desde este momento, han sido muchas y significativas las reformulaciones del concepto de ciudadanía social para construir una definición que incorporase claramente a todos aquellos colectivos tradicionalmente poco visibles en las sociedades occidentales. De la mano de autoras feministas (Pateman 1988, Siim 1997), se ha reivindicado acabar con la invisibilidad de aquellas desigualdades que se producen en el seno del ámbito doméstico y familiar y del ámbito privado, así como de aquellas desigualdades que existen más allá del ámbito mercantil de las relaciones productivas. Nos referimos a mujeres, a personas con enfermedades crónicas o de gran impacto social, a personas migrantes, y entre estos grupos y muchos otros, nos referimos a personas con discapacidad que experimentan trabas materiales y simbólicas para emerger con dignidad del régimen clasificador y estigmatizante que aún existe en nuestra sociedad.

Todos estos grupos de personas se consideran vulnerables por el simple hecho de vivir situaciones que quedan fuera de los estrechos márgenes de la normalidad socialmente construida y aceptada (FOESSA, 1995; Tezanos, 1999). En este sentido, corren el gran riesgo de ver devaluados sus derechos sociales y, por tanto, de ver disminuir las oportunidades de ejercer activamente su ciudadanía. Éste es el trasfondo sociopolítico que ha dado fuerza al discurso de la integración social en el ámbito europeo. La vertiente desiderativa de este discurso pone el énfasis en mantener el valor de la cohesión social entendida como factor de estabilidad e igualdad en las sociedades democráticas. Esto quiere decir que políticamente se refuerza un proyecto de convivencia que promueve la incorporación del mayor número de personas a la normalidad, al ritmo de vida estandarizado de la sociedad.

El debate sobre la integración social, especialmente si lo pensamos para un grupo social como el de las personas con discapacidad, nos 
lleva al debate sobre la normalización. Para ser más precisos, sería necesario hablar de integrar en la normalidad. No son pocas las cuestiones críticas a las que se debe dar respuesta, cuando teórica y metodológicamente se apuesta por esta perspectiva. ¿Integrar y normalizar significa que debemos reducir la complejidad humana a una única forma que consideremos integrador y normal? $\mathrm{O}$, por el contrario, ¿deberemos pensar y actuar en función de la diversidad social y de la pluralidad de opciones? ¿Es preciso plantear vías de integración en la normalidad que tengan prediseñadas las distintas pautas y fases que se deben seguir o es necesario encontrar vías flexibles que permitan cambios de diseño en función de la trayectoria vital de las personas y de las condiciones del contexto social?

Desde la perspectiva de los derechos de ciudadanía la política social se aleja progresivamente de las clasificaciones uniformizantes que años atrás sirvieron para proteger y defender a las personas con discapacidad. Actualmente, la respuesta dirigida a las necesidades de este colectivo tiene como punto de partida la universalidad de los derechos de ciudadanía y de la aspiración del bienestar individual y relacional, pero éste es sólo un punto de partida que puede derivar en un conjunto de alternativas caracterizadas por su pluralidad formal y de contenido. Este planteamiento nos sitúa ante el reto que supone cambiar la mentalidad tradicional de las personas con discapacidad y de las personas sin discapacidades físicas y/o psíquicas sobre las desigualdades sociales y la integración: no se trata de generar medidas o respuestas especiales para garantizar los derechos de unos u otros, sino de pensar en soluciones que faciliten la vida a todos los miembros de la sociedad. Dejemos de pensar en clave de guetos o compartimentos estancos para pensar en una sociedad de personas interdependientes, en que cualquier individuo se presenta con límites y potencialidades, con necesidades no resueltas y con capacidades inexploradas.

Una mirada intensiva a las personas atendiendo a su unicidad y originalidad, una lectura minuciosa y respetuosa de su mundo de relaciones cotidianas y una intervención en el entorno social y físico que neutralice las barreras materiales y simbólicas para el libre desarrollo de la vida permite construir una base teórica y metodológica sólida para comenzar a desarrollar una ciudadanía real y flexible para todos. No podemos afirmar que éste sea un terreno libre de conflictos, sino muy al contrario nos situamos en un espacio presidido por la complejidad y las contradicciones. Pero desde el punto de vista sociopolítico, la defensa reivindicativa de los derechos sociales para las personas con discapacidad parece una estrategia de lo más pertinente para generar, en primer término, prácticas sociales inclusivas y, en segundo término, integradoras. 


\section{INTEGRAR EN LA NORMALIDAD: DE LA PERSPECTIVA OCUPACIONAL A LA PERSPECTIVA DE LA CALIDAD DE VIDA.}

Desde hace mucho tiempo el carácter integrador de las políticas sociales europeas tiene un eco significativo en el conjunto de la sociedad. El cambio de mentalidad respecto a las cuestiones relativas a la desigualdad, la pobreza o la marginación, ha sido un signo de la modernización de nuestra sociedad. Y en este sentido, la construcción del proceso de integración para las personas con discapacidad sigue paralela a las preocupaciones sociales de cada época.

Durante los años de estructuración y consolidación de la democracia española, las políticas públicas y las iniciativas sociales siguiendo las directrices europeas han tenido como prioridad la actuación en el campo del empleo. Desde un punto de vista sociológico, hay una fuerte tradición europea que considera la participación activa en el mercado laboral asalariado como la fuente de emancipación individual y como el recurso central para convertirse en ciudadano de pleno derecho. No en vano la mayoría de los Estados de la Unión Europea (como el Estado español) basan buena parte de los derechos efectivos de la ciudadanía en el estatus de trabajador legalmente contratado. Tal y como nos recuerda Antoni Vilà', el trabajo ocupa un lugar central en nuestra vida y es un elemento clave de integración que ha sido reconocido como derecho social; por ello, desde las Naciones Unidas se establece que los Estados deben reconocer el principio de que las personas con disminución deben estar facultadas para ejercer los derechos humanos, en particular en materia de ocupación. En el contexto de los estados del bienestar, podríamos decir que las medidas desarrolladas de acuerdo con este criterio constituyen un primer salto cualitativo en el reconocimiento del estatuto de ciudadanía de las personas con discapacidad.

Para comprender la centralidad productiva y mercantil que durante estos años ha presidido la formulación del paradigma de la integración, debe considerarse la aceptación pública (gubernamental, sindical y social) del fenómeno del paro estructural y la precariedad laboral en todos los países desarrollados. La devaluación del factor del trabajo asalariado pone en crisis las expectativas de integración y consolidación de derechos de las personas con discapacidad. Si ya en épocas de esplendor económico el mercado ignora las necesidades ocupacionales de colectivos vulnerables, durante una época de reestructuración

Unitat de Promoció i Desenvolupament Comarques Gironines (2000). La inserció laboral de les persones amb disminució a les comarques gironines, Universitat de Girona. 
económica el mercado es altamente impermeable a las necesidades de ocupación de buena parte de la población. Frente a esta situación, la política social europea y estatal ha intensificado su trayectoria de acción positiva hacia todo lo referente a la formación para la ocupación.

En este ámbito, las iniciativas formativas y ocupacionales para las personas con discapacidad han tenido un papel relevante. El esfuerzo realizado desde la iniciativa pública y la privada sin afán de lucro (cooperativas) ha tenido una repercusión considerable en la vida cotidiana de muchas personas jóvenes y adultas con discapacidad. Desde el punto de vista biográfico, el sentimiento de autoestima y de utilidad social ha experimentado una transformación muy significativa. Pero la integración laboral de este colectivo está aún sometida a las resistencias y rigideces económicas, sociales y urbanísticas del entorno. Tal y como pone de manifiesto el estudio sobre "La inserción laboral de las personas con disminución de las comarcas de Girona" (Universitat de Girona, 2000), la gran mayoría de los encuestados se enfrentan a circunstancias que dificultan la inserción laboral, como la falta de puestos de trabajo adaptados, el coste de la adaptación, la existencia de barreras arquitectónicas y sociales, así como la aparición de estereotipos infundados sobre su rendimiento y potencialidades (...) Muchas personas con disminución están suficientemente preparadas para acceder a un puesto de trabajo normalizado, ya sea asalariado, a través de empresas públicas o privadas, o para establecerse como trabajadores autónomos. Incluso con la aplicación de medidas de discriminación positiva dirigidas a paliar las desventajas de este colectivo (cuota de reserva, incentivos para la contratación, subvenciones para establecerse como autónomo, entre otras) los datos nos muestran que estamos lejos de conseguir la plena integración de la persona en el mercado productivo.

Confiar únicamente en la integración por la vía formación y del empleo presenta límites difíciles de superar, incluso considerando las iniciativas públicas. Muchos profesionales dedicados al ámbito de las disminuciones (Jornades de la Fundació Catalana del Síndrome de Down, Barcelona 1999) consideran que ha llegado el momento de dar un segundo salto cualitativo para tratar las dinámicas de integración social de este colectivo. Son muchas las voces de profesionales (Coordinadora de Tallers de Catalunya, 1999) y de asociaciones de padres y madres que reclaman una intervención más integral y dinámica para enfrentarse a las resistencias del entorno. El objetivo de esta reclamación sobrepasa el mundo del trabajo productivo para adentrarse en todas aquellas dimensiones de la vida cotidiana que también permiten que las personas se sientan ciudadanas de pleno derecho. Las dimen- 
siones relativas a la vertiente subjetiva de las personas son las que dan la oportunidad de desarrollar libremente emociones, habilidades, capacidades y deseos para el mismo y para los otros. Sería necesario normalizar lo que se denomina calidad de vida superando la barrera infundada de la discapacidad.

Hablar de calidad de vida significa hablar de la calidad del bienestar que la sociedad está dispuesta a redistribuir entre sus ciudadanos y, por consiguiente, de la igualdad de oportunidades para el acceso a la misma. En una primera aproximación, podemos pensar que la calidad de vida es un concepto claramente definido y consensuado que reúne un sistema de indicadores estándar válido en cualquier contexto y momento histórico. Estamos de acuerdo con la Coordinadora de Tallers cuando manifiesta la validez del término, al mismo tiempo que enfatiza su flexibilidad. Por este motivo existe un acuerdo internacional para defender la utilización del concepto de calidad de vida a partir de un marco de principios amplios y dúctiles -más que de definiciones exactas- que permitan orientar iniciativas bajo una perspectiva plural. Si seguimos la pauta de tales criterios, debemos ser conscientes del esfuerzo (re)constructivo que radica en la naturaleza de este concepto y, en este sentido, de la necesidad de trabajarlo de forma permanente y colectiva. Afirmaríamos que más que a un concepto, la calidad de vida nos aproxima a una metodología de trabajo en equipo en la que las personas con discapacidad, los familiares y los profesionales de los distintos servicios comparten unos objetivos comunes en unos espacios de intervención diferenciados. Tal y como expone la Coordinadora de Tallers de Catalunya, el constructo de la calidad de vida reúne tres perspectivas:

- Por un lado, los indicadores sociales nos permiten valorar los elementos externos que condicionan la calidad de vida material de las personas. Nos referimos a las condiciones de salud, educación, seguridad, vivienda, ocio...

- Por otro, los indicadores psicológicos expresan los aspectos subjetivos que forman parte de la vida y la convivencia. Relaciones e interacciones personales, comunicativas, sociales y cívicas comparten el espacio vivencial junto al nivel de satisfacción que se experimenta en el hogar, en la comunidad, con los amigos, en el ocio y en todo el amplio espectro de la ayuda y el soporte que se recibe y se da día a día.

- Finalmente, las políticas sociales tienen un papel clave a la hora de definir y promover la calidad en todos los servicios que inciden en la vida de las personas con discapacidad y, en general, del conjunto de la ciudadanía. La armonía y el equilibrio entre las 
expectativas y potencialidades de la persona y su materialización supone tener abierto un triple frente de actuación:

- Conocer y atender las demandas del entorno.

- El control de los recursos.

- Las habilidades y la imaginación para satisfacer las demandas.

Como se puede apreciar, la elaboración de las dimensiones que dan cuerpo al constructo de la calidad de vida supera la dicotomía entre lo objetivo y lo subjetivo y se adentra en la articulación de ambos niveles. La pretensión de esta perspectiva es doble: por un lado, quiere responder intelectualmente a la integridad de las vivencias (objetivas y subjetivas) de las personas en la vida cotidiana, y por el otro, quiere dejar constancia de que la calidad de vida, incluso basándose en elementos comunes para el conjunto de las personas, tiene un componente fuertemente idiosincrático, directamente ligado a la originalidad de cada ser humano. Así pues, podemos concluir que nos encontramos ante un proceso de comprensión y acción multidimensional, interactivo y muy dinámico. Individuo, sociedad y desarrollo social son los fundamentos conceptuales de esta perspectiva.

Para hacer operativa y medir la calidad de vida de las personas, sea cual sea su situación personal y social, reproducimos a continuación ocho dimensiones consensuadas entorno a este concepto (Coordinadora de Tallers de Catalunya, 1999):

- Bienestar emocional (incluye el bienestar psicológico).

- Relaciones interpersonales (compendren las relaciones sociales).

- Bienestar material (incluye el trabajo y la seguridad económica).

- Desarrollo personal (relativo a los objetivos y a las competencias y habilidades propias).

- Bienestar físico (incluyendo el tiempo de ocio).

- Autodeterminación (referente al control individual y a la toma de decisiones).

- Inclusión social (comprendiendo la dignidad y el respeto a la propia individualidad).

- Derechos (a la intimidad, a la privacidad, a la posesión y a la responsabilidad cívica).

Una lectura atenta de este conjunto de dimensiones nos permite construir un campo semántico dedicado a la reivindicación y defensa de espacios de visibilidad personal y respeto social para las personas con discapacidad: bienestar, desarrollo, autodeterminación, inclusión y derechos. Todos estos términos necesitan un espacio de expresión que, más allá del lugar de formación o trabajo, se consoliden alrededor de espacios tan cotidianos y básicos para la propia identidad como la vivienda y el hogar, el barrio, los equipamientos y los servicios públicos de la ciudad. 


\section{SOCIALIZARSE PARA LA EMANCIPACIÓN: ESTRATE- GIAS DE APOYO Y DESARROLLO DE LA CREATIVIDAD.}

Los límites sociales que se han impuesto a la libre expresión de las necesidades y de los proyectos de vida de las personas con discapacidad psíquica han generado, en muchos casos, la ruptura de procesos de emancipación personal y de transición hacia una vida autónoma. Las relaciones dentro del ámbito de la familia, de las entidades educativas y ocupacionales no son ajenas a esta inercia.

Se ha de tener en cuenta que el trasfondo cultural de los países mediterráneos sigue manteniendo como pautas emancipadoras la incorporación al mercado laboral y el acceso a una vivienda autónoma (Flaquer, 1998). Estos patrones de orientación tienen como escenario ideal la salida del hogar familiar cuando, además de tener trabajo, se tiene pareja y vocación de formar una familia con hijos e hijas. Estos dos ejes coinciden con los dos grandes obstáculos sociales que presiden la trayectoria de vida de las personas con discapacidad: disfrutar de un trabajo remunerado en igualdad de condiciones respecto al resto de personas trabajadoras y tener la posibilidad y la libertad de establecer relaciones afectivas de pareja. Además es importante señalar que el encarecimiento de los precios de las viviendas y la insignificante proporción de viviendas sociales limita drásticamente cualquier deseo de emancipación. Si trabajo y pareja quedan todavía fuera del ámbito del desarrollo personal y social de las personas con discapacidad, y si a ello añadimos la rigidez de la oferta del mercado inmobiliario, no es, pues, atrevido afirmar que la decisión de salir del hogar familiar para vivir en una vivienda autónoma bajo una pluralidad de fórmulas ( individual, en pareja, con amigos o amigas, en colectividad residencial, etc. ) es prácticamente inexistente y que, cuando surge, recibe más reticencias que respuestas favorables.

Por este motivo, salir del núcleo familiar como proceso que permite desarrollar la identidad hacia la madurez y la autonomía, la capacidad de cuidarse a sí mismo y a los demás y las responsabilidades hacia la comunidad de vecinos y ciudadanos, toma un protagonismo destacado. Este proceso no debe entenderse únicamente vinculado a una necesidad material básica y universal, sino ligado a necesidades de carácter simbólico para generar autoestima y conciencia de futuro y de mejora.

Mientras leemos esta líneas, pueden ser muchas las personas que piensen que estos planteamientos sólo son pertinentes si nos encontramos ante casos con grados leves de discapacidad. Querríamos llamar la atención del lector sobre la cantidad de experiencias, muchas de ellas entorno a una pluralidad de ofertas residenciales en el Estado 
Español, Europa y EEUU ${ }^{2}$ que, con el apoyo de las diferentes administraciones locales y estatales, permiten dar una oferta de calidad a cualquier persona con discapacidad (independientemente de su grado), favoreciendo así su capacidad de expresión y sus habilidades. Es necesario, pues, desarrollar una red de recursos públicos basada en una estructura de apoyos profesional y flexible.

¿Qué queremos decir cuando nos referimos a una estructura de apoyo profesional y flexible? Para responder a esta pregunta, previamente, hemos de definir, la palabra apoyo para pasar, a continuación, a delimitar los ámbitos en los cuales ha de desarrollarse. Utilizamos el término apoyo como consecuencia lógica de la defensa que hacemos de un paradigma que considera que:

- Las personas somos sujetos con capacidades y limitaciones.

Las capacidades de desarrollo y adaptación de las personas son tan diversas y plurales como diversas y plurales son ellas mismas.

- El proceso de desarrollo como individuos y sujetos sociales está fuertemente condicionado por las relaciones interpersonales $y$ las condiciones ambientales (sociales y urbanísticas) del entorno.

- Disfrutar de bienestar y calidad de vida es un proceso de convivencia basado en la interdependencia y la reciprocidad: las personas aportan habilidades y bienes emocionales a la vez que reciben ayudas materiales y emocionales de los otros.

En definitiva, dar apoyo significa acompañar a las personas a lo largo de su ciclo de vida para facilitarles el acceso a aquellos recursos que les darán dignidad como seres humanos y seguridad y libertad para desarrollar su propio proyecto biográfico. Una estructura de apoyo profesional implica también la ayuda y seguimiento de calidad dinamizado por un equipo de personas con conocimientos y experiencia en el ámbito de la discapacidad. Una estructura flexible de apoyos comporta una metodología de trabajo que sea tan dinámica y cambiante como lo es el propio ciclo de vida de la persona, en estrecha relación con el contexto socioeconómico, cultural e histórico que la envuelve.

2 Entre muchas iniciativas desarrolladas en Catalunya, destacamos aquellas que las investigadoras conocen de primera mano: los pisos asistidos para personas con un nivel medio de discapacidad de la cooperativa Aspasim en Barcelona, o los pisos asistidos del Taller Auria Cooperatiu (TAC) en Igualada. En Europa, la guía de buenas prácticas del Programa Helios II de la Unión Europea destaca el proyecto de la asociación Herefordshire Lifestyle y First Key Partnership, en el Reino Unido; la Comunità e Cooperativa Progetto Sud, en Italia; el Proyecto Marjala, en Finlandia y la asociación británica de automovilistas Shopmobility. Good for people and towns, también en el Reino Unido. 
A partir de una estructura de apoyos, las administraciones, que cuidan los derechos y la calidad de vida de las personas con discapacidad, pueden desarrollar fórmulas de implicación más ligadas a las propuestas elaboradas por las propias personas con discapacidad, por los profesionales y por las asociaciones de familiares. Esta es una oportunidad que, a pesar de no ser siempre sencilla de llevar a cabo, ayuda a construir un espacio de colaboración y participación más fluido entre ciudadanía y poderes públicos. Es un paso cualitativo hacia la democracia participativa, que permite acercar la política social a las necesidades reales de la población y que reconoce la validez de las aportaciones e iniciativas de la comunidad para dinamizar la plena integración de las personas con discapacidad.

\section{REFLEXIONES FINALES.}

Pensar en términos de calidad de vida nos lleva a integrar tanto las dimensiones objetivas de las condiciones de vida como las dimensiones más claramente subjetivas. Sentimientos, emociones, aspiraciones, sueños se incorporan como elementos clave para el análisis y la intervención social.

Si damos un protagonismo especial al ámbito de la vida subjetiva de las personas, una de las líneas más importantes y complejas que se habría de desarrollar sería la que hace referencia al tratamiento de las transiciones biográficas de las personas con discapacidad psíquica. Es bien conocido de todos los profesionales que los momentos personales de cambio para este colectivo son momentos especialmente vividos desde la ignorancia, desde la soledad, desde el conflicto. Por ello es tan necesario reconocer una línea pedagógica y de socialización que prepare a las personas con discapacidad psíquica para una vida plural, dinámica y cambiante.

En definitiva, este entramado conceptual responde a la necesidad de dar un salto cualitativo en el ámbito teórico y en el ámbito sociopolítico, es decir, un cambio en la manera de comprender y actuar en el ámbito de la discapacidad. En el momento en que se rompe la legitimidad política de segregar e incluso, de hacer invisibles a las personas con discapacidad, se inicia todo un proceso de reflexión que avanza sobre sus propios pasos. Ésta es una conquista que reúne el esfuerzo y la experiencia de muchas personas que tienen alguna discapacidad, de sus familiares y de un conjunto de profesionales muy comprometidos con las capacidades de desarrollo de las personas. Actualmente asistimos a un proceso conflictivo porque se vuelve a cuestionar aquello que, supuestamente, había generado un significativo consenso; nos re- 
ferimos, por un lado, al reconocimiento de los derechos humanos como base para garantizar los derechos sociales de todas las personas sin distinción de ningún tipo, tampoco por razón de discapacidad física o psíquica; $y$, por otro, al abandono progresivo de la perspectiva estrictamente médicobiologista de la discapacidad.

Desde esta perspectiva los factores medioambientales, es decir, las condiciones y las formas de operar del medio físico, social, económico, cultural y político, pasan a considerarse fuentes promotoras de la discapacidad. Es necesario, pues, cambiar las condiciones de este medio tan plural, y hacerlo relativizando el concepto de discapacidad. Esto significa dejar de hablar de discapacidad y hablar de las estrategias que sirven para capacitar. Quiere decir también que es necesario hablar de cada individuo desde su originalidad y huir, por tanto, de las descripciones que uniformizan un colectivo de personas muy diversas entre sí.

Casi se podría hablar del derecho a la capacidad o, dicho de otra manera, del derecho a desarrollar las capacidades. Bajo esta perspectiva no hay una conciencia ilimitada de las capacidades humanas, bien al contrario, éste es sólo un punto de partida para ser conscientes de las limitaciones que cualquier persona tiene para afrontar la vida. Es por eso que, frente a los factores de límite, se defiende una respuesta basada en las relaciones sociales. La interacción con los otros, es decir, el ámbito de la interdependencia, se incorpora cada vez más en los discursos de la política social como estrategia de respuesta frente a las necesidades o aspiraciones concretas de las personas con discapacidad. Este salto cualitativo ha tenido como consecuencia pasar de la perspectiva de la independencia a la perspectiva relacional.

Desde esta perspectiva reivindicamos todos aquellos espacios ${ }^{3}$ de relaciones sociales de proximidad y confianza como espacios para la comunicación y el intercambio, como medios de aprendizaje y de producción de conocimientos, como centros de recursos donde poder escoger, como fuentes de calidad de vida. Pero no podemos obviar que todo lo que hemos expuesto hasta ahora representa más una estructura utópica que una realidad de facto. Aún queda mucho por hacer y muchos espacios sociales por conquistar para poder hablar de un ejercicio real de los derechos de ciudadanía de las personas con discapacidad psíquica. Por este motivo pensamos que es más pertinente defender, en primer lugar, el derecho a la inclusión social, es decir, el derecho a poder decidir, a ser visible en cualquier espacio y actividad tanto de la vida pública como de la vida privada. 
Uno de los campos que, previsiblemente, traerá más innovaciones en la producción del bienestar de las personas con discapacidad psíquica es el que relaciona las diversas actividades de la vida. No nos referimos a actividades de ocio, sino a todos aquellos aspectos que se desarrollan día a día y que tienen que ver con el tiempo de vida que no pasa por la escuela, el centro de formación o el trabajo; en definitiva, nos referimos al tiempo de las relaciones familiares, de amistad, de vecindad, de aficiones u obligaciones domésticas, etc. Estos espacios tienen en común su elevado valor de uso. Por eso es muy interesante pensar en cómo utilizarlos más y mejor.

Con estas reflexiones, que en ningún caso son una propuesta cerrada, querríamos invitar al lector a liberar un espacio personal para dejarse sorprender cuando piense o intervenga en el ámbito de las discapacidades abriendo una ventana a la utopía, una oportunidad para que 'los otros' demuestren lo que son capaces de hacer y de crear. En definitiva, es sólo una cuestión de justicia.

\section{BIBLIOGRAFÍA}

AIGUABELLA, J. y GONZÁLEZ, M.: Análisis de necesidades de personas adultas con grave discapacidad. Ministerio de Trabajo y Asuntos Sociales, Madrid, 1996.

ÁNGEL, A.: Hacia una sociedad ambiental. Editorial El Labrador, Bogotá, 1995.

ARENDT, H.: La condición humana. Paidós, Barcelona,1993.

CARBONELL, E. et al: El constructe de la qualitat de vida. Els DIPS a Catalunya davant el Segle XXI. Coordinadora de Tallers per a Minusvàlids Psíquics de Catalunya, Barcelona, 1999.

CASTELLS, M.: La era de la información: Economía, sociedad y cultura. Vol.2: El poder de la identidad, Alianza Editorial, Madrid, 1998.

Diari Oficial de la Generalitat de Catalunya: "De l'estructura del Sistema Català de Serveis Socials", Núm.1997-13.1.1995.

FEAPS APPS: Congrés Nacional de Families de Persones amb Retard Mental. Reunions i Ciència, S.L. Barcelona, 2000.

FLAQUER, L.: El destino de la familia, Ariel, Barcelona, 1998.

FOESSA: Informe sociológico sobre la situación social en España, Fundación FOESSA, Madrid, 1995.

Instituto Nacional de Servicios Sociales: La discapacidad en el siglo XXI. Plan de acción. Una propuesta de futuro, Ministerio de Trabajo y Asuntos Sociales, Madrid, 1997. 
LEMKOV, L.; TEJERO, E.; TORRABADELLA, L.: Costos biogràfics en la lluita pel benestar, E. Mediterrània, Barcelona, 2000.

MARSHALL, T.H.y BOTTOMORE, T.: "Ciudadanía y clases sociales. Cuarenta años después", Revista Española de Investigaciones Cientificas 79, 1997, p. 297-344.

PALLISERA, M. (coord.): Atenció a persones adultes amb discapacitat psíquica, Universitat de Girona, Girona, 1997.

SCOTT, A.: Helios II Guía Europea de Buena Práctica. Hacia la Igualdad de Oportunidades de las Personas Discapacitadas, Oficina de Publicaciones Oficiales de las Comunidades Europeas, Luxemburgo, 1996.

TEZANOS, J.F. (ed.): Tendencias en desigualdad y exclusión social, Editorial Sistema. Madrid, 1999.

VERDUGO, M.A; DE BORJA, F.; DE URRÍES, J. (coord.): Hacia una nueva concepción de la discapacidad, Amarú, Salamanca, 1999.

Vicerectorat de Docència i Estudiants de la Universitat de Girona: La inserció laboral de les persones amb disminució a les comarques gironines, Universitat de Girona, Girona, 2000. 